\title{
Teaching Fractions in a Course Basic College Mathematics
}

\author{
Anastasiya Yakunina, Ph.D. \\ Associate Professor of Mathematics \\ Voorhees College, \\ 213 Wiggins Drive, Denmark, SC 29042 USA \\ ayakunina@voorhees.edu
}

\begin{abstract}
The article is dedicated to the problem of optimization of teaching Basic College Mathematics in a college. An author's original method of teaching fractions is presented. Concrete instructions for increasing effectiveness of teaching fractions are given. Special attention is paid to a student-centered approach that is considered in this article as an effective instrument for an improvement of the results of teaching Basic Mathematics at college level. All conclusions and recommendations are based on the results of modern research in Education and on the author's teaching experience (12 years).
\end{abstract}

\section{Key Words}

Basic college mathematics, teaching fractions, methods for increasing effectiveness of teaching math in college, student-centered education

Basic College Mathematics courses are designed for students who don't demonstrate satisfactory mathematical skills on entry-level college placement tests.

The goal of these courses is to help students acquire the necessary math skills for taking more advanced math courses required for their major.

But the instructor may face several serious barriers from the outset. First, students may have studied this material (whole numbers, fractions, etc.) in middle school and high school but still don't understand the concepts. Students often resent having to take these courses again in college. Perhaps the most significant barrier to learning is student dislike of math in general. At the outset of each semester, I ask students whether they like mathematics or not. The results have been the same each year-about $90 \%$ of students say they don't like math because it's difficult and boring.

After reviewing a diverse variety of sources on the problem, and based on my personal classroom experiences, I have created an original "decomposition" method for teaching fractions that seems to alleviate some of the learning barriers mentioned previously.

At the start of his/her unit on fractions, the instructor should give students a clear definition of prime numbers (a natural number greater than 1 whose factors are 1 and the 
number itself) and a definition of a composite number (a number which can be "decomposed" as a product of prime numbers). It is essential to discuss several concrete math problems at this point.

Problem 1. Determine whether the following numbers are prime numbers or composite numbers. If the number is composite, find its prime factors.
a. 5
b. 12

Discussion Strategy:

For (a), ask your students "Can the number 5 be decomposed (represented as a product of other numbers)? Students will answer "No, this number can't be decomposed." So the students have identified 5 as a prime number. It can't be divided.

For (b), ask your students "Can the number 12 be decomposed (represented as the product of other numbers?). Students will answer "Yes it can," recognizing that 12 can be divided into 2 times 6 . You should write $12=2 \times 6$ on the board. Say to the students, "So we've decomposed 12 but is $2 \times 6$ a final answer? Or can we continue the process of decomposition? Remember that the answer must contain only prime numbers. Are all the factors here prime? Students should answer "No, it's not a final answer because 6 is not a prime number; it can be decomposed."

Write $12=2 \times 6=2 \times 2 \times 3$ on the board. At this point, ask if the process of decomposition is complete. "Are all the numbers prime?" Students should say yes.

There may be some students who will ask if 12 can be represented as $4 \times 3$, instead of $2 \times 6$. Both variants are correct and decompose is the same:

$12=4 \times 3=2 \times 2 \times 3 \quad 12=2 \times 6=2 \times 2 \times 3$

It's very important to solve a number of problems like these with the students joining in the conversation. They need to master decomposition of numbers because it helps them see the structure of numbers and to solve problems that contain fractions quite easily,

It's not necessary to ask your students to memorize the definitions of a prime and a composite number because practice shows that students intuitively understand these concepts. Almost all students can tell a composite number from a prime number even if they don't remember the exact definitions. Practice is a key to success here. You'll need to solve enough problems with your students to make sure that everybody understands how to decompose 
numbers. Only after you see that your students can do it correctly and easily, can you begin to study fractions and how to cancel a common factor in a numerator and denominator.

The normal method for teaching students how to cancel a common factor is by finding the greatest common factor (GCF) of a numerator and denominator of the fraction [4]. But the author knows from experience that it's difficult for the students to memorize the algorithm of finding GCF and they often confuse GCF with LCM (the least common multiple). Actually finding GCF is not necessary to simplify a fraction. It's enough just to decompose the numerator and denominator and then to cancel all common factors.

Before discussing a concrete example, it's important to remind students that the numerator is the "top" of a fraction and the denominator is the "bottom" of a fraction in order to make the material more understandable for all students in the class.

Problem 2. Simplify this fraction into its simplest form): $\frac{20}{24}$

Discussion Strategy: The instructor should tell the students to "Decompose the numerator (the "top" of the fraction) and the denominator (the "bottom" of the fraction) writing this on the board:

$$
\frac{20}{24}=\frac{10 \cdot 2}{6 \cdot 4}
$$

You should point out that "The process of decomposition is incomplete," and ask students to continue decomposing the numbers until all factors become prime numbers. Write this on the board:

\section{$\frac{5 \cdot 2 \cdot 2}{2 \cdot 3 \cdot 2 \cdot 2}$}

At this point, you can say "Now all factors are prime and we can cancel common factors (here we can cancel 2 twice)" writing this on the board:

$$
\frac{5 \cdot 2 \cdot 2}{2 \cdot 3 \cdot 2 \cdot 2}=\frac{5}{2 \cdot 3}
$$

You can now tell the students "We've canceled all common factors so it's time to "assemble" our fraction)" writing this on the board: 
This is the final answer. Since we've canceled the common factors, our fraction is in its simplest form.

Here is another problem demonstration to write on the board:

$\frac{36}{210}=\frac{4 \cdot 9}{21 \cdot 10}=\frac{2 \cdot 2 \cdot 3 \cdot 3}{3 \cdot 7 \cdot 5 \cdot 2}($ we can cancel 2 and 3$)=\frac{2 \cdot 3}{7 \cdot 5}=\frac{6}{35}$

You can see that to solve such problems, students don't really need a concept of GCF. They just need to decompose the numerator and denominator, cancel common factors and simplify the resulting fraction.

After students master this material, the lessons can move on to multiplication and division of fractions. (Usually instructors study addition and subtraction first but decomposition works perfectly for multiplication and division of fractions too. Practicing it with multiplication and division will help students gain confidence and the skills necessary for studying more difficult material (addition and subtraction of fractions).

Problem 3. Solve the following multiplication of fractions: (Write this on the board):

$\frac{18}{35} \cdot \frac{21}{32}=\frac{2 \cdot 9}{7 \cdot 5} \cdot \frac{7 \cdot 3}{8 \cdot 4}$

Remind your students that all numbers in the expression must be decomposed completely before we can cancel common factors.

$\frac{2 \cdot 3 \cdot 3}{7 \cdot 5} \cdot \frac{7 \cdot 3}{2 \cdot 2 \cdot 2 \cdot 2 \cdot 2}($ we can cancel 2 and 7$)=\frac{3 \cdot 3 \cdot 3}{5 \cdot 2 \cdot 2 \cdot 2 \cdot 2 \cdot 2}=\frac{27}{80}$.

The same principle works great for division of fractions. You just need to give your students this formula for division:

$\frac{a}{b} \div \frac{c}{d}=\frac{a}{b} \cdot \frac{d}{c}=\frac{a d}{b c}$

Using the formula,

$\frac{36}{45} \div \frac{24}{42}=\frac{36}{45} \cdot \frac{42}{24}=\frac{6 \cdot 6}{9 \cdot 5} \cdot \frac{6 \cdot 7}{8 \cdot 3}=\frac{2 \cdot 3 \cdot 2 \cdot 3}{3 \cdot 3 \cdot 5} \cdot \frac{2 \cdot 3 \cdot 7}{2 \cdot 2 \cdot 2 \cdot 3}$

Tell your students we can cancel three twos and two threes so the final answer is $\frac{7}{5}\left(=1 \frac{2}{5}\right)$.

Practice shows that students solve such problems correctly, easily, and quickly when they use the described method and they usually enjoy the process of decomposing numbers and cancelling common factors. 
Addition and subtraction of fractions are much more difficult for students.

As known, fractions can be added and/or subtracted only if they have a common denominator. If the denominators are different, the students need to find a common denominator and reduce the fractions to this denominator. As a rule, instructors tell the students that they need to find LCM (the least common multiple) of the denominators in order to find a common denominator of the fractions [4]. The algorithm of finding LCM seems difficult and complicated to many students and they often confuse it with the algorithm of finding GCF.

With this in mind, it can be more effective to use decomposition of denominators of both fractions to find the common denominator. Before solving the problems by this method the students need to be reminded that a numerator and denominator of a fraction can be multiplied by the same non-zero number without changing the value of the fraction: $\frac{a}{b}=\frac{a \cdot c}{b \cdot c}$

So the procedure of adding fractions will look like this:

Problem 4. Solve this problem: $\frac{7}{10}+\frac{1}{6}$

Discussion: Tell students "The denominators are different and we can't add the fractions right now. Let's decompose the both denominators to see their structure" and write this on the board $\frac{7}{5 \cdot 2}+\frac{1}{3 \cdot 2}$

Say to students "The denominators must be the same in order to add fractions. What is missing in the denominator of the first fraction? We have 5 and 2 there but we don't have 3. So, to get the missing number 3 in the "bottom" of the first fraction, we need to multiply the "top" and the "bottom" of the fraction by 3." Write this on the board:

\section{$\frac{7 \cdot 3}{5 \cdot 2 \cdot 3}$}

"Now, what is missing in the "bottom" of the second fraction?" Students will say "We have 3 and 2 there but we don't have 5."

Ask "How can we get 5 in there?"

Students will say, "We need to multiply the 'top' and the 'bottom' of the fraction by 5."

$$
\frac{1}{3 \cdot 2}=\frac{1 \cdot 5}{3 \cdot 2 \cdot 5}
$$


Now we have:

$$
\frac{7}{10}+\frac{1}{6}=\frac{7}{5 \cdot 2}+\frac{1}{3 \cdot 2}=\frac{7 \cdot 3}{5 \cdot 2 \cdot 3}+\frac{1 \cdot 5}{3 \cdot 2 \cdot 5}
$$

Say to the students "Now the denominators are the same $(5 \cdot 2 \cdot 3=3 \cdot 2 \cdot 5)$, so we're in the position to add our fractions. But before doing it we need to simplify our fractions (we don't need the prime factorizations of the bottoms anymore because we've already found the common denominator):

$$
\frac{7 \cdot 3}{5 \cdot 2 \cdot 3}+\frac{1 \cdot 5}{3 \cdot 2 \cdot 5}=\frac{7}{30}+\frac{5}{30}=\frac{12}{30} \quad\left(=\frac{2}{5}\right)
$$

Problem 5. Solve this problem: $\frac{3}{16}+\frac{5}{8}$.

Discussion: tell the students "The denominators are different and we can't add the fractions right now. Let's decompose the both denominators to see their structure" and write this on the board:

$$
\frac{3}{2 \cdot 2 \cdot 2 \cdot 2}+\frac{5}{2 \cdot 2 \cdot 2}
$$

Say to the students "The denominators must be the same in order to add fractions. What is missing in the denominator of the first fraction?"

Students will say "Nothing is missing, we have four twos here."

Say to students "What is missing in the denominator of the second fraction?"

Students will say "Two is missing because in the bottom of the first fraction we have four twos and in the bottom of the second fraction we have only three twos. So we need to multiply the top and the bottom of the second fraction by the missing number 2 ".

Now we have:

$$
\frac{3}{16}+\frac{5}{8}=\frac{3}{2 \cdot 2 \cdot 2 \cdot 2}+\frac{5}{2 \cdot 2 \cdot 2}=\frac{3}{2 \cdot 2 \cdot 2 \cdot 2}+\frac{5 \cdot 2}{2 \cdot 2 \cdot 2 \cdot 2}=\frac{3}{16}+\frac{10}{16}=\frac{13}{16} .
$$

Of course I've presented only a brief description of teaching fractions by decomposition, but I hope this method will resonate with other Basic Mathematics teachers who face similar barriers to student success.

I'd like to conclude with a few suggestions for my colleagues that have worked for me. 
First, it is critical to maintain student engagement - even for those students who claim to know "all this stuff." A good solution to this problem is to make such students your helpers during a class session. For example, you can ask them to "remind" you of some useful formulas instead of writing them down by yourself. Actually every student in class can become your "helper" for solving more or less difficult math problems. Of course even the best students sometimes make errors so you should be ready to correct them if needed. It's of the utmost importance to keep your students willing to assist you during the class, to make them actively involved in the learning process. It's possible to attain this only if you are polite and friendly. When you encourage students to help and they make an error, you should never criticize them rudely and don't allow other students in class to do so. Instead, correct the student politely and at the end of the discussion thank the student for his/her help.

In accordance with the student-centered theory of teaching, maintaining a friendly, comfortable atmosphere during every class session is one of the essential factors that will allow you to succeed in teaching not only Basic Mathematics but courses in all disciplines.

\section{References}

1. Friedberg, Solomon. Teaching Mathematics in Colleges and Universities: Case Studies for Today's Classroom/Solomon Friedberg. - Student ed. Conference Board of Mathematical Sciences, Rhode Island, 2001

2. Kelton, Suzanne. An Introduction to Teaching Mathematics at the College Level/ Suzanne Kelton - Assumption College (https://www.ams.org/profession/careerinfo/grad-school/Kelton-TEACH.pdf)

3. Krantz, Steven. How to Teach Mathematics/Steven G. Krantz - $3^{\text {rd }}$ edition. American Mathematical Society, Rhode Island, 2015, P. 26.

4. Aufman, Richard and Lockwood, Joanne. Introductory Algebra: an Applied Approach, 2011 